\title{
Produção do conhecimento em enfermagem acerca do empoderamento em situações crônicas de saúde
}

\section{Knowledge production in nursing about empowerment in chronic health conditions \\ Producción del conocimiento en enfermería en cuanto al empoderamiento de las condiciones de salud crónicas}

\author{
Lina Márcia Miguéis Berardinelli'; Nathália Aparecida Costa Guedes ${ }^{I I}$; Araci Carmen Clos ${ }^{\text {III }}$; \\ Juliana Pereira Ramos ${ }^{I V}$; Anna Carolina dos Santos Chaves ${ }^{\mathrm{V}}$; Clícia Vieira ${ }^{\mathrm{VI}}$
}

\begin{abstract}
RESUMO: Este estudo teve por objetivo analisar a produção científica na área da enfermagem sobre o empoderamento em situações crônicas de saúde. Realizou-se revisão integrativa para responder à questão: Como se apresenta a literatura em enfermagem acerca do empoderamento das pessoas em situações crônicas de saúde? A coleta de dados ocorreu em fevereiro e março de 2015, nas bases de dados Public/Publish Medline (PUBMED), Scientific Eletronic Library Online (SCIELO) e Biblioteca Virtual de Saúde (BVS) de modo integrado. A busca de artigos foi realizada com as palavras-chave combinadas: empoderamento, enfermagem e doença crônica, nas referidas bases de dados e nos idiomas Inglês, Português e Espanhol. Foram selecionadas 12 publicações na íntegra, do período de 2006 a 2015. Emergiram duas categorias: O empoderamento em situações crônicas de saúde; e o conceito de empoderamento e a capacitação dos profissionais. É necessário ampliar a produção científica nacional e internacional sobre a temática abordada.

Palavras-Chave: Empoderamento; doença crônica; enfermagem; capacitação profissional.
\end{abstract}

\begin{abstract}
This study aimed to analyze scientific production in the nursing field on the subject of empowerment in chronic health conditions. An integrative review was conducted to answer the question: What is the state of the nursing literature about empowering people with chronic health conditions? Data was collected in an integrated manner from the Public/Publish Medline (PubMed), Scientific Electronic Library Online (SciELO) and Virtual Health Library (BVS) databases in February and March 2015. The search for articles was conducted in those databases with the combined keywords 'empowerment', 'nursing' and 'chronic disease' in English, Portuguese and Spanish. Twelve complete publications from 2006 to 2015 were selected. Two categories emerged: empowerment in chronic health situations; and the concept of empowerment and professional training. The national and international scientific literature on the subject needs to be enlarged.

Keywords: Empowerment; chronic illness; nursing; professional training.
\end{abstract}

RESUMEN: Este estudio tuvo como objetivo analizar la producción científica en el área de enfermería en cuanto al empoderamiento en situaciones crónicas de salud. Se ha realizado una revisión integradora para responder a la pregunta: ¿Cómo se presenta la literatura en enfermería en cuanto al empoderamiento de las personas en situaciones crónicas de salud? La recolección de datos se llevó a cabo en febrero y marzo de 2015, en las bases de datos Public/Publish Medline (PubMed), Scientific Electronic Library Online (SciELO) y la Biblioteca Virtual en Salud (BVS) de una manera integrada. La búsqueda de artículos se llevó a cabo con las palabras clave combinadas: empoderamiento, enfermería y enfermedad crónica, en las mencionadas bases de datos y en los idiomas Inglés, Portugués y Español. Se han seleccionado 12 publicaciones en su totalidad, del período de 2006 a 2015. Han surgido dos categorías: El empoderamiento en situaciones crónicas de salud; y el concepto de empoderamiento y la formación profesional. Es necesario ampliar la producción científica nacional e internacional sobre el tema abordado. Palabras Clave: Empoderamiento; enfermedad crónica; enfermería; formación profesional.

\section{INTRODUÇÃO}

O conceito empowerment e a reflexão sobre o seu significado pedagógico assumem um papel de destaque no panorama das doenças crônicas não transmissíveis
(DCNT). As situações crônicas de saúde têm sido desafiadoras para a humanidade, em razão do seu crescimento em todos os continentes e, com, isso

\footnotetext{
IEnfermeira. Professora Adjunta, Programa de Pós-Graduação da Faculdade de Enfermagem da Universidade do Estado do Rio de Janeiro. Bolsista do Programa de Incentivo à Produção, Técnica e Artística (Prociência). Brasil. E-mail: 1.m.b@uol.com.br.

"Enfermeira. Mestranda do Programa de Pós-Graduação da Faculdade de Enfermagem da Universidade do Estado do Rio de Janeiro. Brasil. E-mail: nathyyguedes@gmail.com.

IIIEnfermeira. Mestre em Filosofia. Professora Assistente da Faculdade de Enfermagem da Universidade do Estado do Rio de Janeiro. Editora Científica da Revista de Enfermagem UERJ. Brasil. E-mail: araciclos@yahoo.com.br.

IV Aluna do $7^{\circ}$ Período do Curso de Graduação da Faculdade de Enfermagem da Universidade do Estado do Rio de Janeiro. Bolsista de Iniciação Científica, Conselho Nacional de Desenvolvimento Científico e Tecnológico. Rio de Janeiro, Brasil. E-mail: julianapereiraramos@gamil.com.

${ }^{\vee}$ Enfermeira. Mestranda do Programa de Pós-Graduação da Faculdade de Enfermagem da Universidade do Estado do Rio de Janeiro. Brasil. E-mail: carolchavesrj@gmail.com.

VIEnfermeira. Mestranda do Programa de Pós-Graduação da Faculdade de Enfermagem da Universidade do Estado do Rio de Janeiro. Brasil. E-mail: cliciavieira@gmail.com.
} 
exigindo mudança nas práticas de saúde, no sentido de repensar novas formas de abordagens e acompanhamento que ultrapassem o modelo vigente, biologicista e organicista, que tem sido insuficiente para atender às demandas e à complexidade que envolvem o cuidado às pessoas com DCNT.

Os indicadores epidemiológicos da Organização Mundial de Saúde (OMS) demonstram que as DCNT constituem um problema de saúde de grandes proporções, pois têm correspondido a $72 \%$ das causas de óbito. Atualmente, são responsáveis por $60 \%$ de todo o ônus decorrente de doenças no mundo. E alerta-se que, em 2020, serão responsáveis por $80 \%$ da carga de doença dos países em desenvolvimento ${ }^{1}$.

Da mesma forma, as consequências dos transtornos gerados pelas DCNT são múltiplas, as quais trazem prejuízos à saúde das pessoas, impedindo-as de viverem plenamente e de realizarem uma série de atividades do ponto de vista profissional e social. As estimativas para o Brasil sugerem que a perda de produtividade no trabalho e a diminuição da renda familiar resultante gerada por elas levarão a uma perda na economia brasileira de US\$ 4,18 bilhões entre 2006 e 20151,2.

Para tanto, a Organização Pan-americana de Saúde e a OMS ressaltaram que as possíveis intervenções nas DCNT podem ser, por exemplo, ações educativas voltadas para a mudança de comportamentos, assim como cuidados continuados, de longa duração, planejados de forma prospectiva, proativa e apoiados fundamentalmente na atenção primária à saúde. A capacitação de pacientes é fundamental, de modo a desenvolver sua adesão, com apoio das famílias, a metas de cuidado, bem como autonomia na solução de problemas e participação nos processos decisórios clínicos, sempre sobre supervisão das equipes de saúde ${ }^{2}$.

Corroborando tais concepções, as linhas de ação da Carta de Ottawa e da Declaração de Alma Ata reafirmam que é necessário intensificar as ações das estratégias de promoção em saúde no cotidiano dos serviços, promover a autonomia das pessoas, indivíduos e profissionais, para que, em conjunto, possam compreender a saúde como resultante das condições de vida, propiciando um desenvolvimento social mais equitativo ${ }^{3}$.

Diante disso, as intervenções direcionadas aos clientes devem investir nas ações de autocuidado, conhecimento e acesso a informações como imprescindíveis para a tomada de decisão, desenvolver as habilidades sociais, visando às práticas de empowerment, isto é, empoderamento ${ }^{4}$.

O empoderamento tem assumido significações que se referem ao desenvolvimento de potencialidades, aumento de informação e percepção, com a finalidade de promover uma participação real e simbólica, que possibilite a democracia ${ }^{5}$.
O empoderamento permite a aprendizagem dialógica, desenvolvimento da consciência crítica, na qual o indivíduo encontra sentido para um modo de viver saudável próprio/autônomo/personalizado. Somente a informação, compartilhada nos processos educativos, por si só não modificará os comportamentos em relação à situação-problema. Embora o conhecimento seja uma condição necessária para que ocorra o processo de mudança de certa prática ou comportamento, outras variáveis como a atitude terão de ser mudadas para que determinado comportamento nocivo seja modificado ${ }^{6}$.

Este trabalho pretende contribuir para o desenvolvimento da produção do conhecimento científico em enfermagem, sobre o empoderamento das pessoas acometidas por doenças crônicas, e para a atualização de enfermeiros, provocando reflexões para mudanças no cuidado em saúde, além de potencializar novos objetos de pesquisas. Portanto, o objetivo deste estudo foi analisar a produção científica na área de enfermagem sobre o empoderamento de pessoas em situações crônicas de saúde.

\section{Metodologia}

Trata-se de um estudo de revisão integrativa, método relevante para a enfermagem, pois seus profissionais são constantemente desafiados a buscar conhecimento científico, com objetivo de promover melhoria no cuidado. A prática baseada em evidências encoraja a utilização de resultados de pesquisas na assistência à saúde prestada, nos diferentes níveis de atenção, corroborando a importância da pesquisa no exercício clínico? .

A revisão integrativa promove a apresentação crítica das produções científicas, facilitando a leitura por parte dos profissionais que não dispõem de tempo para realizar a leitura integral do material disponível. Dessa forma, por meio deste método, é possível analisar os estudos já publicados que levam a conclusões gerais a respeito de uma determinada área de estudo ${ }^{8}$.

Assim, para delineamento do estudo, foram seguidos os seis passos metodológicos descritos por Ganong: seleção das hipóteses ou das questões para a revisão; estabelecimento dos critérios para a seleção da amostra; apresentação das características da pesquisa primária; análise dos dados; realização da interpretação dos resultados e, por último, a apresentação da revisão ${ }^{9}$.

Na primeira etapa, foi delimitada a questão para a revisão: Como se apresenta a literatura em enfermagem acerca do empoderamento das pessoas com doenças crônicas? A seguir foram estabelecidos os critérios de seleção dos estudos, sendo estes: artigo completo, disponível online, periódico qualificado, resultados de pesquisas, relatos de experiência, revisões e 
atualidades, nos idiomas Português, Inglês ou Espanhol disponíveis em meio eletrônico, no recorte temporal dos últimos 10 anos (2005-2015). Foram excluídas do estudo publicações não disponíveis na íntegra e que se afastassem do tema proposto para a pesquisa.

A coleta de dados observou as seguintes características primárias: a produção dos dados foi realizada em fevereiro e março de 2015, a partir das bases de dados Public/Publish Medline (PUBMED), Scientific Eletronic Library Online (SCIELO) e Biblioteca Virtual de Saúde (BVS) de modo integrado com as bases: Base de Dados de Enfermagem (BDENF), Literatura Latino-Americana e do Caribe em Ciências da Saúde (LILACS), Medical Literature Analysis and Retrieval System Online (MEDLINE). A busca de artigos foi processada com as palavras-chave combinadas: empoderamento, enfermagem e doença crônica, nas referidas bases de dados e nos idiomas inglês, português e espanhol.

O estudo de revisão integrativa, como instrumento da prática baseada em evidências, caracteriza-se por ser uma abordagem que se direciona tanto para o cuidado clínico quanto para o ensino fundamentado no conhecimento e na constatação da qualidade da prática clínica ${ }^{8}$.

As evidências são classificadas de forma hierárquica para a avaliação de pesquisas ou outras fontes de informação, baseando-se na categorização da Agency for Healthcare Research and Quality (AHRQ) dos Estados Unidos da América. A sua classificação abrange seis níveis, descritos a seguir: nível 1 metanálise de múltiplos estudos controlados; nível 2 - estudo individual com desenho experimental; nível 3 - estudo com desenho quase experimental como estudo sem randomização com grupo único pré e pós-teste, séries temporais ou caso-controle; nível 4 - estudo com desenho não experimental como pesquisa descritiva correlacional e qualitativa ou estudos de caso; nível 5 - relatório de casos ou dado obtido de forma sistemática, de qualidade verificável ou dados de avaliação de programas; nível 6 - opinião de autoridades respeitáveis baseada na competência clínica ou opinião de comitês de especialistas, incluindo interpretações de informações não baseadas em pesquisas; opiniões reguladoras ou legais ${ }^{8}$.

O instrumento de coleta de dados constava dos seguintes itens para registrar as informações extraídas dos artigos: título, autores, periódico publicado, resumo, tipo de pesquisa/ metodologia utilizada, principais resultados e conclusão. $\mathrm{O}$ tratamento dos dados foi aplicado no material coletado, com base na análise temática de Minayo, que estabelece as etapas: préanálise com leitura e releitura do material, exploração do material obtido e tratamento dos resultados com organização, interpretação e apresentação dos resultados na forma de categorias ${ }^{10,11}$.

Os resultados foram discutidos à luz da literatura pertinente ao tema ${ }^{12-30}$.

\section{Resultado e Discussão}

Na base de dados SCIELO, foi encontrada uma publicação científica, a partir da associação dos descritores citados. Na base de dados PUBMED foram localizados 23 artigos, sendo que somente 10 estavam disponíveis e versavam sobre a temática escolhida. Foram identificados 12 artigos na base de dados BVS, sendo que somente dois deles estavam disponíveis na íntegra e se aproximavam da temática escolhida. Totalizaram, portanto, 12 artigos $^{12-23}$.

Dessa forma, pôde-se perceber um número reduzido de artigos científicos publicados sobre a temática do empoderamento em clientes com DCNT, nas bases pesquisadas.

Os artigos encontrados e os respectivos autores, periódico, base de dados e tipos de pesquisa estão relacionados na Figura 1.

No que se refere ao ano de publicação, verificase um número de textos maior nos anos de 2014 e 2013, ambos com quatro estudos cada, seguidos por dois em 2008, um em 2006 e um em 2010. Não houve produção sobre o tema em 2005. Quanto à autoria dos trabalhos publicados, um artigo foi escrito por enfermeiros e médicos e 11 somente por enfermeiros.

Em relação ao delineamento metodológico da pesquisa, nas bases de dados e biblioteca virtual consultadas, verifica-se o predomínio de pesquisas quantitativas, em seis artigos; seguindo-se pesquisas qualitativas, em quatro textos; um estudo teórico e um de atualidades.

Os delineamentos da pesquisa e seus níveis de evidência são mostrados na Figura 2.

Os resultados gerados demonstram que as pesquisas sobre o empoderamento nas doenças crônicas se apresentam em número reduzido, possivelmente por ser considerado, ainda, um campo a ser explorado.

Diante do material selecionado, foi realizada análise temática, após a leitura exaustiva dos artigos. A análise teve como objetivo organizar e sumarizar os dados para responder ao problema proposto neste estudo. E a interpretação buscou associar o descrito nos artigos com conhecimentos mais amplos, já obtidos sobre o assunto ${ }^{12}$. A partir de então, da proximidade entre os artigos e a repetição dos temas entre eles, emergiram duas categorias: $\mathrm{O}$ empoderamento em situações crônicas de saúde e $\mathrm{O}$ conceito de empoderamento e a capacitação dos profissionais. Essas categorias são tratadas a seguir.

\section{O empoderamento em situações crônicas de saúde}

O empoderamento nas situações crônicas de saúde foi a temática de maior destaque entre os trabalhos analisados, mantendo-se presente durante todo o recorte temporal estudado. 


\begin{tabular}{|c|c|c|c|c|}
\hline Título & Autores & $\begin{array}{c}\text { Periódico } \\
\text { (volume, ano) }\end{array}$ & $\begin{array}{c}\text { Base de } \\
\text { dados }\end{array}$ & $\begin{array}{l}\text { Tipo de } \\
\text { pesquisa }\end{array}$ \\
\hline $\begin{array}{l}\text { Barriers to and factors } \\
\text { facilitating empowerment in } \\
\text { elderly with COPD. }\end{array}$ & $\begin{array}{l}\text { Fotoukian Z. Shahboulaghi } \\
\text { FM, Khoshknab MF, } \\
\text { Mohammadi E }{ }^{12}\end{array}$ & $\begin{array}{l}\text { Med J Islam } \\
\text { Repub Iran.v.28 } \\
\text { 2014; }\end{array}$ & PUBMED & $\begin{array}{l}\text { Pesquisa } \\
\text { qualitativa }\end{array}$ \\
\hline $\begin{array}{l}\text { Effect of family } \\
\text { empowerment on the quality } \\
\text { of life of school-aged } \\
\text { children with asthma }\end{array}$ & $\begin{array}{l}\text { Payrovee Z, Kashaninia Z, } \\
\text { Alireza Mahdaviani S, } \\
\text { Rezasoltani } \mathrm{P}^{13}\end{array}$ & $\begin{array}{l}\text { Tanaffos. v. } 13 . \\
2014\end{array}$ & PUBMED & $\begin{array}{l}\text { Pesquisa } \\
\text { qualitativa }\end{array}$ \\
\hline $\begin{array}{l}\text { Effect of family } \\
\text { empowerment model on } \\
\text { quality of life in children } \\
\text { with chronic kidney diseases }\end{array}$ & $\begin{array}{l}\text { Ghazavi Z, Minooei MS, } \\
\text { Abdeyazdan Z, Gheissari A }{ }^{14}\end{array}$ & $\begin{array}{l}\text { Iran J Nurs } \\
\text { Midwifery Res. v. } \\
\text { 19. } 2014\end{array}$ & PUBMED & $\begin{array}{l}\text { Pesquisa } \\
\text { qualitativa }\end{array}$ \\
\hline $\begin{array}{l}\text { Concept analysis of } \\
\text { empowerment in old people } \\
\text { with chronic diseases using } \\
\text { a hybrid model }\end{array}$ & $\begin{array}{l}\text { Fotoukian Z, Shahboulaghi FM, } \\
\text { Khoshknab MF, Mohammadi } \\
\text { E }^{15}\end{array}$ & $\begin{array}{l}\text { Asian Nurs Res. v. } \\
\text { 8. } 2014\end{array}$ & PUBMED & $\begin{array}{l}\text { Pesquisa } \\
\text { qualitativa }\end{array}$ \\
\hline $\begin{array}{l}\text { Validity and reliability of } \\
\text { Korean version of health } \\
\text { empowerment scale (K-HES) } \\
\text { for older adults }\end{array}$ & Park C, Park $\mathrm{YH}^{16}$ & $\begin{array}{l}\text { Asian Nurs Res. } \\
\text { v.7. } 2013\end{array}$ & PUBMED & $\begin{array}{c}\text { Pesquisa } \\
\text { quantitativa }\end{array}$ \\
\hline $\begin{array}{l}\text { Integrative health coach } \\
\text { training: a model for shifting } \\
\text { the paradigm toward patient- } \\
\text { centricity and meeting new } \\
\text { national prevention goals }\end{array}$ & $\begin{array}{l}\text { Smith LL, Lake NH, Simmons } \\
\text { LA, Perlman A, Wroth S, } \\
\text { Wolever RQ }\end{array}$ & $\begin{array}{l}\text { Glob Adv Health } \\
\text { Med. v. 2. } 2013\end{array}$ & PUBMED & $\begin{array}{c}\text { Pesquisa } \\
\text { quantitativa }\end{array}$ \\
\hline $\begin{array}{l}\text { Can participation in } \\
\text { documentation influence } \\
\text { experiences of involvement } \\
\text { in care decision-making? }\end{array}$ & Vestala $\mathrm{H}$, Frisman $\mathrm{GH}^{18}$ & $\begin{array}{l}\text { Open Nurs J. v. } 7 . \\
2013\end{array}$ & PUBMED & $\begin{array}{c}\text { Projeto } \\
\text { experimental, } \\
\text { randomizado }\end{array}$ \\
\hline $\begin{array}{l}\text { Rural women, technology, } \\
\text { and self-management of } \\
\text { chronic illness. }\end{array}$ & $\begin{array}{l}\text { Weinert C, Cudney S, Hill } \\
\text { WG }^{19}\end{array}$ & $\begin{array}{l}\text { Can J Nurs Res. } \\
\text { v. } 40.2008\end{array}$ & $\begin{array}{l}\text { PUBMED } \\
\text { MEDLINE }\end{array}$ & $\begin{array}{c}\text { Pesquisa } \\
\text { quantitativa }\end{array}$ \\
\hline $\begin{array}{l}\text { Empowerment interventions, } \\
\text { knowledge translation and } \\
\text { exchange: perspectives of } \\
\text { home care professionals, } \\
\text { clients and caregivers }\end{array}$ & $\begin{array}{l}\text { St-Cyr Tribble D, Gallagher F, } \\
\text { Bell L, Caron C, Godbout P, } \\
\text { Leblanc J, Morin P, Xhignesse } \\
\text { M, Voyer L, Couture } \mathrm{M}^{20}\end{array}$ & $\begin{array}{l}\text { BMC Health Serv } \\
\text { Res. v.8 } 2008\end{array}$ & $\begin{array}{l}\text { PUBMED } \\
\text { MEDLINE }\end{array}$ & $\begin{array}{c}\text { Pesquisa } \\
\text { quantitativa e } \\
\text { qualitativa }\end{array}$ \\
\hline $\begin{array}{l}\text { Influence of a computer } \\
\text { intervention on the } \\
\text { psychological status of } \\
\text { chronically ill rural women: } \\
\text { preliminary results }\end{array}$ & Hill W, Weinert C, Cudney S21 & $\begin{array}{l}\text { Nurs Res.v.55. } \\
2006\end{array}$ & $\begin{array}{l}\text { PUBMED } \\
\text { MEDLINE }\end{array}$ & $\begin{array}{c}\text { Pesquisa } \\
\text { quantitativa }\end{array}$ \\
\hline $\begin{array}{l}\text { Análise conceitual de } \\
\text { autogestão do indivíduo } \\
\text { hipertenso }\end{array}$ & $\begin{array}{l}\text { Balduino, A.F.A; Mantovani, } \\
\text { M.F; Lacerda, M.R; Meier, M.J.2 }\end{array}$ & $\begin{array}{l}\text { Rev Gaúcha } \\
\text { Enferm. v.34. } \\
2013\end{array}$ & $\begin{array}{l}\text { SCIELO, } \\
\text { BVS }\end{array}$ & $\begin{array}{l}\text { Estudo teórico- } \\
\text { revisão }\end{array}$ \\
\hline $\begin{array}{l}\text { Empowerment das famílias } \\
\text { de crianças dependentes de } \\
\text { tecnologia }\end{array}$ & $\begin{array}{l}\text { Leite, N.SL; Cunha, SR; } \\
\text { Tavares, MFL }{ }^{23}\end{array}$ & $\begin{array}{l}\text { Rev enferm Uerj, } \\
\text { v.192010 }\end{array}$ & BVS & $\begin{array}{l}\text { Artigo de } \\
\text { atualidade }\end{array}$ \\
\hline
\end{tabular}

FIGURA 1: Descrição dos estudos incluídos na revisão integrativa, segundo título, autor, periódico (ano, volume), base de dados e tipo de pesquisa. 2006-2014.

O empoderamento é visto com uma forma de aumentar o compromisso, a autogestão, melhorando a eficácia e qualidade de vida e podendo reduzir os custos de invalidez e de cuidados na doença. É um processo de capacitação com foco na capacidade de participar conscientemente nos cuidados de saúde, na tomada de decisões e está associado com a consciência das próprias forças e habilidades de desenvolvimento, autoestima, bem-estar e autocapacidade ${ }^{12}$. Tal concepção valoriza a autonomia conforme dispõe a literatura ${ }^{23-30}$.

Os artigos descreveram adultos e idosos em situações crônicas de saúde, como sujeitos do estudo, em que o empoderamento foi objeto central para reforçar o potencial deles para o aprendizado e o uso das ferra- 


\begin{tabular}{|c|c|c|}
\hline Título & Delineamento da pesquisa & Nível de evidência \\
\hline $\begin{array}{l}\text { Barriers to and factors facilitating empowerment in } \\
\text { elderly with COPD }{ }^{12}\end{array}$ & Estudo qualitativo & 4 \\
\hline $\begin{array}{l}\text { Effect of Family Empowerment on the Quality of life of } \\
\text { School-Aged Children with Asthma }{ }^{13}\end{array}$ & Estudo qualitativo & 4 \\
\hline $\begin{array}{l}\text { Effect of family empowerment model on quality of life in } \\
\text { children with chronic kidney diseases }{ }^{14}\end{array}$ & Estudo qualitativo & 4 \\
\hline $\begin{array}{l}\text { Concept analysis of empowerment in old people } \\
\text { with chronic diseases using a hybrid model }\end{array}$ & Estudo qualitativo & 4 \\
\hline $\begin{array}{l}\text { Validity and reliability of Korean version of } \\
\text { health empowerment scale (K-HES) for older adults' }\end{array}$ & Estudo quantitativo & 4 \\
\hline $\begin{array}{l}\text { Integrative Health Coach Training: A Model for Shifting } \\
\text { the Paradigm Toward Patient-centricity and Meeting } \\
\text { New National Prevention Goals }{ }^{17}\end{array}$ & Estudo quantitativo & 4 \\
\hline $\begin{array}{l}\text { Can participation in documentation influence } \\
\text { experiences of involvement in care decision-making? }{ }^{18}\end{array}$ & $\begin{array}{c}\text { Estudo qualitativo e } \\
\text { quantitativo }\end{array}$ & 4 \\
\hline $\begin{array}{l}\text { Rural women, technology, and self-management } \\
\text { of chronic illness. }{ }^{19}\end{array}$ & Estudo quantitativo & 4 \\
\hline $\begin{array}{l}\text { Empowerment interventions, knowledge translation and } \\
\text { exchange: perspectives of home care professionals, } \\
\text { clients and caregivers }{ }^{20}\end{array}$ & $\begin{array}{c}\text { Estudo qualitativo e } \\
\text { quantitativo }\end{array}$ & 4 \\
\hline $\begin{array}{l}\text { Influence of a computer intervention on the } \\
\text { psychological status of chronically ill rural women: } \\
\text { preliminary results }{ }^{21}\end{array}$ & Estudo quantitativo & 4 \\
\hline $\begin{array}{l}\text { Análise conceitual de autogestão do indivíduo } \\
\text { hipertenso }{ }^{22}\end{array}$ & Estudo teórico- revisão & 5 \\
\hline $\begin{array}{l}\text { Empowerment das famílias de crianças dependentes de } \\
\text { tecnologia }{ }^{23}\end{array}$ & Estudo atualidades & 5 \\
\hline
\end{tabular}

FIGURA 2: Descrição dos estudos incluídos na revisão integrativa segundo o delineamento de pesquisa e nível de evidências. 2006-2014.

mentas de que necessitam para viver de forma tão independente quanto possível e para manter/melhorar a sua própria qualidade vida e da sua família ${ }^{20}$. Para motivar esse comportamento para saúde, observaram-se várias estratégias, como uso de tecnologias - computador, internet -, grupos de discussão, questionários ${ }^{12,15,16,18-20}$.

Percebeu-se também que os artigos, segundo esta categoria, versaram sobre o empoderamento das crianças e famílias com doença crônica, considerando tal intervenção a melhor estratégia de controle da patologia e de sua gestão pelas crianças e seus pais. Nesse processo, os pacientes já não desempenham um papel passivo no curso da doença. Em vez disso, eles envolvem-se ativamente no decorrer do tratamento, assumindo a responsabilidade de cuidar de si mesmos, com conhecimento e habilidades apropriadas a esse respeito e, consequentemente, diminuindo a internação e as crises ${ }^{13,14}$. Essas mudanças comportamentais refletem os princípios da autonomia desejada ${ }^{23-30}$.

\section{O conceito de empoderamento e a capaci- tação dos profissionais}

O interesse e atração para o conceito de empowerment foi estimulado, em meados de 1980, em conjunto com a concepção de promoção da saúde da OMS, como um processo de capacitação da comunidade para aumentar e melhorar habilidades para o autocuidado e manutenção da saúde15. De acordo com os artigos, o conceito de empoderamento está ligado à participação ativa, compromisso para a mudança, conhecimento para resolução de problemas, responsabilidade para o autocuidado, competência do cliente e controle da saúde ou da vida15,21,22. O empoderamento em saúde promove a capacidade de participar conscientemente das decisões e dos cuidados de saúde. É a promoção da autonomia e da cidadania ${ }^{23-30}$.

O papel dos profissionais de saúde é facilitar o engajamento do paciente em direção a seus objetivos de saúde, utilizando a melhor metodologia disponível para motivá-lo ${ }^{17}$. A participação do profissional de saúde, principalmente o enfermeiro, pode influenciar a tomada de decisão clínica, com o objetivo de obter tratamento adequado. Para facilitar essa participação, é preciso informá-lo sobre o autocuidado necessário, utilizando estratégias para envolvê-lo. É necessário que as preferências do paciente sejam levadas em consideração para mantê-lo no processo de cuidado18.

Os profissionais de saúde precisam reconhecer as necessidades dos pacientes, o nível de conhecimento sobre a sua patologia e suas expectativas com o tratamento e, a partir daí, buscar um comportamento modificado e comprometido com a sua saúde e seu autocuidado. 


\section{Considerações Finais}

Diante do objetivo inicialmente proposto, constatou-se que a produção sobre a temática empoderamento em situações crônicas de saúde, no recorte temporal de 2006 a 2015 foi reduzida, totalizando 12 trabalhos, agrupados em duas categorias: $\mathrm{O}$ empoderamento nas situações crônicas de saúde e o conceito de empoderamento e a capacitação dos profissionais. Ressalta-se a insuficiência de trabalhos na língua portuguesa.

É fundamental que as políticas públicas atendam às necessidades dessa população, com incentivos a programas de promoção da saúde que valorizem o empoderamento do indivíduo, com o objetivo de melhorar sua qualidade de vida.

É necessário que a produção científica nacional e internacional sobre a temática abordada seja ampliada.

\section{REFERÊNCIAS}

1.Ministério da Saúde (Br). Plano de ações estratégicas para o enfrentamento das doenças crônicas não transmissíveis (DCNT) no Brasil 2011-2022. 2011. [citado em 05 mar 2015]. Disponível em: portal.saude.gov.br/portal/ arquivos/pdf/cartilha_plano.pdf.

2.Organização Pan-Americana da Saúde; Organização Mundial da Saúde. Doenças crônicas não transmissíveis: estratégias de controle e desafios e para os sistemas de saúde. Brasília (DF) 2011. [citado em 05 mar 2015]. Disponível em: http://apsredes.org/site2012/wp-content/ uploads/2012/06/Condicoes-Cronicas_flavio1.pdf.

3.Teixeira MB. Empoderamento de idosos em grupos direcionados à promoção da saúde. [dissertação de mestrado] Rio de Janeiro: Escola Nacional de Saúde Pública; 2002. [citado em 05 mar 2015]. Disponível em: http://portalteses.icict.fiocruz.br/transf.php?id $=000034$ $\& \operatorname{lng}=\mathrm{pt} \& \mathrm{nrm}=$ iso\&script $=$ thes_cover.

4.Otero LM, Zanetti ML, Teixeira CRS. Características sociodemográficas e clínicas de portadores de diabetes em um serviço de atenção básica à saúde. Rev Latino-Am Enfermagem. 2007; [citado em 05 mar 2015] Disponível em: http://www.scielo.br/pdf/rlae/v15nspe/pt_08.pdf.

5.Wendhausen ALP, Barbosa TM, Borba MC. Empoderamento e recursos para a participação em conselhos gestores. Rev Saúde Sociedade. 2006; [citado em 05 mar 2015]: 15(3) 131-144. Disponível em:http://www.scielo. br/scielo.php?pid $=$ S010412902006000300011\&script $=$ sci_arttext.

6.C̄arvalho GS. Literácia para a saúde: um contributo para a redução das desigualdades em saúde. In: Leandro M, Araújo M, Costa M, organizadores. Saúde: as teias da discriminação social. Braga $(\mathrm{Pt})$ : Universidade do Minho; 2002, 119-35.

7.Galvão CM, Sawada NO, Mendes IAC. A busca das melhores evidências. Rev esc enferm USP [Internet]. 2003; 37: 43-50. [citado em 02 fev 2015]. Disponível em: http://www.scielo.br/pdf/reeusp/v37n4/05.pdf
8.Souza MT, Silva MD, Carvalho, R. Revisão integrativa: o que é e como fazer [Internet]. 2010; [citado em $02 \mathrm{fev}$ 2015] 8:102-6. Disponível em: http://apps.einstein.br/revista/arquivos/PDF/1134-Einsteinv8n1_p102106_port.pdf 9. Ganong LH. Integrative reviews of nursing research. Res Nurs Health. 1987; 10 (1):1-11. Acess for EBSCO host ${ }^{\circledR}$. 10.Minayo MCS. O desafio do conhecimento - pesquisa qualitativa em saúde. 11 ${ }^{\mathrm{a}}$ ed. São Paulo: Hucitec; 2008. 11.Minayo MCS. Organizadora. Pesquisa social: teoria, método e criatividade. 20a ed. Petrópolis (RJ): Vozes; 2002. 12.Fotoukian Z. Shahboulaghi FM, Khoshknab MF, Mohammadi E. Barriers to and factors facilitating empowerment in elderly with COPD. Med J Islam Repub Iran. 2014; 2015; 28:155 [cited in 2015, Feb 06]. Available from: http://www.ncbi.nlm.nih.gov/pmc/articles/PMC4322331/ 13.Payrovee Z, Kashaninia Z, Alireza Mahdaviani S, Rezasoltani P. Effect of family empowerment on the quality of life of School-Aged Children with Asthma. Tanaffos. 2014; [cited in 2015 Feb 03] 13: 35-42. Available from: http://www.ncbi.nlm.nih.gov/pmc/articles/PMC4153273/ 14.Ghazavi Z, Minooei MS, Abdeyazdan Z, Gheissari A. Effect of family empowerment model on quality of life in children with chronic kidney diseases. Iran J Nurs Midwifery Res. 2014; 19: 371-5. [cited in 2015 Feb 03]. 19 (4): 371-5. Available from: http://www.ncbi.nlm.nih. gov/pmc/articles/PMC4145491/

15.Fotoukian Z, Shahboulaghi FM, Khoshknab MF, Mohammadi E. Concept analysis of empowerment in old people with chronic diseases using a hybrid model. Asian Nurs Res (Korean Soc Nurs Sci). 2014; [cited in 2015 Feb 03] 8 (2): 118-127. Available from: http://www.asian-nursingresearch. com/article/S1976-1317(14)00021-8/abstract

16.Park C, Park YH. Validity and reliability of Korean version of health empowerment scale (K-HES) for older adults. Asian Nurs Res (Korean Soc Nurs Sci). 2013; [cited in 2015 Feb 03] 7 (3): 142-8. Available from: http://www.asian-nursingresearch.com/article/S19761317(13)00040-6/abstract

17.Smith LL, Lake NH, Simmons LA, Perlman A, Wroth $S$, Wolever RQ. Integrative health coach training: a model for shifting the paradigm toward patient-centricity and meeting new national prevention goals. Glob Adv Health Med 2013; [cited in 2015 Feb 03] 2(3): 66-74. Available from: http://www.ncbi.nlm.nih.gov/pmc/articles/PMC3833534/

18.Vestala H, Frisman GH. Can participation in documentation influence experiences of involvement in care decision-making? Open Nurs J 2013; [cited in 2015 Feb 03] 7: 66-72. Available from: http://www.ncbi.nlm.nih. gov/pmc/articles/PMC3680981/.

19. Weinert C, Cudney S, Hill WG. Rural women, technology, and self-management of chronic illness. Can J Nurs Res. 2008; [cited in 2015 Feb 03] 40: 114-34. Available from: http://www.ncbi.nlm.nih.gov/pubmed/18947095 20.St-Cyr Tribble D, Gallagher F, Bell L, Caron C, Godbout P, Leblanc J, et al. Empowerment interventions, knowledge translation and exchange: perspectives of home care professionals, clients and caregivers. BMC Health Serv Res. 2008; [cited in 2015 Feb 03] 8:177. Available from: http://www.ncbi.nlm.nih.gov/pmc/articles/PMC2528009/ 
21.Hill W, Weinert C, Cudney S. Influence of a computer intervention on the psychological status of chronically ill rural women: preliminary results. Nurs Res 2006; [cited in 2015 Feb 03] 55 (1): 34-42. Available from: http:// www.ncbi.nlm.nih.gov/pmc/articles/PMC1484522/

22.Balduino AFA, Mantovani MF; Lacerda MR; Meier MJ. Análise conceitual de autogestão do indivíduo hipertenso. Rev Gaúcha Enferm 2013; [citado em 03 fev 2015] 34(4). Disponível em: http://dx.doi.org/10.1590/ S1983-14472013000400005

23.Leite NSL, Cunha SR, Tavares MFL. Empowerment das famílias de crianças dependentes de tecnologia. Rev enferm UERJ. 2011; 19:152-6.

24.Vasconcelos EM. O poder que brota da dor e da opressão: empowerment, sua historia, teoria e estratégias. São Paulo: Paulus; 2011.

25.Carvalho SR. Os múltiplos sentidos da categoria empowerment no projeto de promoção da saúde. Cad Saúde Publica. 2004; [citado em 10 abr 2015] 20:1088-95. Dis- ponível em: http://www.scielo.br/scielo.php?script=sci arttext\&pid=S0102-311X2004000400024

26.Ferreira MS, Castiel LD. Que empowerment, qual promoção da saúde? Convergências e divergências conceituais em práticas preventivas em saúde. Cad Saúde Publica. 2009; [citado em 10 abr 2015] 25: 68-76. Disponível em: http://www.scielo.br/scielo.php?pid=S0102311X2009000100007\&script $=$ sci arttext

27.Dunst CJ, Trivette CM, Deal AG. Enabling \& empowering families: principles $\&$ guidelines for practice. Cambridge (MA): Brookline Books; 2010.

28.Labonte R. Health promotion and empowerment: reflections on professional practice. Health education guartely. 1994 [cited in 2015 Apr 10] 21: 253-68. Avaliable from: http://www.ncbi.nlm.nih.gov/pubmed/8021151 29.Freire P. Pedagogia da autonomia: saberes necessários à pratica educativa. 43 a ed. Rio de Janeiro: Paz e Terra; 2013. 30.Freire P. Pedagogia do oprimido. $17^{\mathrm{a}}$ ed. Rio de Janeiro: Paz e Terra; 2011. 